\title{
Editorial: Accomplishments, Collaborative Projects and Future Initiatives in Breast Cancer Genetic Predisposition
}

\author{
Paolo Peterlongo ${ }^{1 *}$ and Luis G. Carvajal-Carmona ${ }^{2,3,4}$ \\ ${ }^{1}$ Genome Diagnostics Program, IFOM The FIRC Institute for Molecular Oncology, Milan, Italy, ${ }^{2}$ Genome Center, University of \\ California, Davis, Davis, CA, United States, ${ }^{3}$ Department of Biochemistry and Molecular Medicine, School of Medicine, \\ University of California, Davis, Sacramento, CA, United States, ${ }^{4}$ Population Sciences and Health Disparities Program, \\ University of California Davis Comprehensive Cancer Center, Sacramento, CA, United States
}

Keywords: breast cancer genetic predisposition, GWAS, VUS, PRS, splicing

\section{Editorial on the Research Topic}

Accomplishments, Collaborative Projects and Future Initiatives in Breast Cancer Genetic Predisposition

Since the discovery of breast cancer genes BRCA1 and BRCA2 (BRCA1/2) over two decades ago, much has been accomplished in the field of breast cancer genetic predisposition. On one hand, novel genes harboring rare pathogenic variants, most of which act in the same BRCA1/2 pathway, causing increasing disease risk have been identified. In addition, several single nucleotide polymorphisms (SNPs) that modify the breast cancer risk in individuals with BRCA1/2 mutations

\section{OPEN ACCESS}

Edited and reviewed by:

Giuseppe Giaccone,

Georgetown University, United States

*Correspondence:

Paolo Peterlongo

paolo.peterlongo@ifom.eu

Specialty section:

This article was submitted to

Cancer Genetics,

a section of the journal

Frontiers in Oncology

Received: 05 June 2019

Accepted: 15 August 2019

Published: 28 August 2019

Citation:

Peterlongo $P$ and

Carvajal-Carmona LG (2019) Editorial: Accomplishments, Collaborative

Projects and Future Initiatives in Breast Cancer Genetic Predisposition.

Front. Oncol. 9:841. doi: 10.3389/fonc.2019.00841 are now known. These moderate-to-high penetrant genetic variants now represent key elements for improving risk prediction in familial cases. On the other hand, hundreds of common low-risk SNPs have been discovered and can be incorporated into prediction models to improve the identification of women at risk of breast cancer in the general population. Moreover, multifactorial analyses, family studies and high throughput functional assays have been developed to validate candidate genes, classify the variants of uncertain significance (VUS) detected by gene-panel next generation sequencing in clinical and research settings, and to measure the risk magnitude conferred by known pathogenetic variants. Articles in the present Frontiers in Oncology e-book explore these aspects of breast cancer predisposition further.

Individuals who carry $B R C A 1 / 2$ pathogenic variants have an average cumulative risk of developing breast cancer, by age 80 years, of $\sim 70 \%$ (1). Thanks to the efforts of the collaborators of the PALB2 Interest Group (http://www.palb2.org/), PALB2 is now considered the third high-risk gene with pathogenic variants associated with $44 \%$ lifetime risk of developing breast cancer (2). The moderate-penetrance genes $A T M$ and CHEK2 are also associated with breast cancer, conferring a $20 \%$ average lifetime risk $(3,4)$. More recently, BARD1, RAD51D, BRIP1, and RAD51C have been proposed as risk factors for triple-negative breast cancer [TNBC; (5)], indicating that the risk associated with pathogenic variants in each gene may vary by tumor subtype. Support for this hypothesis is the latest emerging breast cancer gene FANCM which has also shown to confer a higher risk for TNBC (6-8). All these genetic factors explain only about half of the familial cases, hence novel breast cancer genes or alleles are yet to be detected (9). In this e-book, the impact of BRCA1/2 mutations and of novel genes was investigated in unexplored populations and in breast cancer progression. Solano et al. studied the BRCA1/2 mutation spectra in high-risk Ashkenazi Jewish population from Argentina. They reported that, in addition to carriers of known Ashkenazi 
founder mutations, up to $7 \%$ of tested individuals were positive for other $B R C A 1 / 2$ pathogenic variants. In a second study, Torrezan et al. performed whole exome sequencing in Brazilian breast cancer probands that were negative for causal variants in most known predisposition genes. Beside a very rare and novel pathogenic variants in ATM and BARD1, respectively, the authors found rare and possibly damaging variants in several candidate genes. Tang et al. aimed at the identification of genes associated with the progression of breast cancer. The authors developed a free-scale gene coexpression networks to explore associations between gene sets and clinical features, and to identify candidate biomarkers. Breast cancer is not exclusively a female disease and about $1 \%$ of all cases arise in males. As reviewed by Rizzolo et al., 13\% of male breast cancer (MBC) are due to pathogenic variants in BRCA2 while CHEK2 and PALB2 account for a smaller proportion of cases. In their study these authors suggest that monoallelic mutation of MUTYH gene, which cause the recessive MUTYH-associated polyposis (MAP) syndrome may also cause MBC.

The identification of common SNPs associated with breast cancer risk and of those that modify the breast cancer risk in individuals with $B R C A 1 / 2$ pathogenic variants is the most significant success of the Breast Cancer Association Consortium (BCAC, http://bcac.ccge.medschl.cam.ac.uk/) and of the Consortium of Investigators of Modifiers of BRCA1/2 (CIMBA, http://cimba.ccge.medschl.cam.ac.uk/). A meta-analysis of genome wide association studies (GWAS), which combined data from 122,977 breast cancer cases and 105,974 controls, resulted in the identification of a total of 172 risk-associated SNPs explaining about half of the familial relative risk [the risk of first-degree relatives of breast cancer patients of developing the disease; (10)]. Ten additional SNPs were found by the analyses of breast cancer cases with estrogen receptor (ER) negative tumors (11), bringing the total number of known breast cancer SNPs to 182 SNPs. While individually these risk factors are not clinically relevant, they can be combined into polygenic risk scores (PRS) that can be predictive of cancer risk in BRCA1/2 mutation carriers and in the general population $(1,12)$. As discussed in this e-book, in a review by Rivandi et al., many of these GWAS-identified SNPs are located outside coding regions and are tags for mostly unknown, causal or functional variants. Hence, their identification would provide better estimates of the explained familial relative risk, thereby improving polygenetic PRSs and increase our understanding of the biological mechanisms involved in breast cancer susceptibility. The success of BCAC and CIMBA in identifying several low risk alleles, resides in the capability of coordinating the efforts of over 180 worldwide groups or studies contributing DNA samples and data from breast cancer cases and control and from BRCA1/2 mutation carriers. The French Genetic Modifiers of BRCA1 and BRCA2 (GEMO) Group, described in this e-book by Lesueur et al., is one of the larger studies within CIMBA. GEMO was initiated in 2006 and today involves 32 clinics and 17 diagnostics laboratories that, as of April 2018, collected 5,303 participants.
As discussed above, many variants in BRCA1/2 and in other established or candidate breast cancer genes have uncertain clinical significance. These VUSs, which are typically rare missense variants, represent a serious clinical problem as carrier risk estimates are often unclear. The Evidence-based Network for the Interpretation of Germline Mutant Alleles (ENIGMA; https://enigmaconsortium.org) was formed to determine the clinical significance of variants in $B R C A 1 / 2$ and other known or suspected breast cancer genes (13). To this aim, ENIGMA gathers pathologists, epidemiologists, geneticists, bio-informaticians, genetic counselors, and molecular biologists into working groups to assess the clinical relevance of variants by applying statistical approaches and multifactorial likelihood models, studying tumor markers, or performing functional assays. Two articles in this e-book provide insights into VUSs classification. The first study, by Zuntini et al., investigated whether co-segregation analyses, integrated with functional data and in silico predictions, could improve VUSs interpretation and counseling in carrier families. In the second study, Caleca et al., used an in vitro assay specifically designed to test the BRCA2 and PALB2 gene products interaction and showed initial pathogenicity evidence for two very rare missense variants in these genes. A special class of VUS are those suspected to cause mRNA splicing defects. One of the ENIGMA working groups was established to improve the clinical classification of likely spliceogenic variants. Members of this working group contributed articles exploring some of the aspects of this variant class. For example, Fraile-Bethencourt et al. identified eight spliceogenic variants in exon 16 of BRCA2 by minigene assay, highlighting the efficiency of this approach for clinical classification. In silico tools for splicing defect prediction may play a key role in VUS analysis. Moles-Fernández et al. used 99 in vitro-validated variants to evaluate the performance of six commonly used splicing in silico tools. Finally, Farber-Katz et al. conducted analyses of BRCA1/2 variants using a novel RNA-massively parallel sequencing assay capable to perform quantitative and qualitative analysis of transcripts. Similarly, Lattimore et al. utilized targeted RNAseq to re-assess BRCA1/2 mRNA isoform expression patterns in lymphoblastoid cell lines. Recommendations from these two studies will facilitate the application of targeted RNA-seq approaches for the quantitative characterization of $B R C A 1$ and $B R C A 2$ germline splicing alterations.

In summary, articles in the present e-book move the field of breast cancer genetics in several aspects, ranging from characterizing genetic variation in new populations to developing and applying tools for variant re-classification. Since the discovery of the role of $B R C A 1 / 2$ on breast cancer risk, much has been learned and through the tremendous international, multiand inter-disciplinary efforts of consortia such as BCAC, CIMBA, and ENIGMA. The next decade promises to illuminate many new aspects of breast cancer risk prevention on families and in the general population.

\section{AUTHOR CONTRIBUTIONS}

PP and LC-C wrote this manuscript. 


\section{ACKNOWLEDGMENTS}

We are grateful to all authors that contributed to this e-book and to Nandita Mitra for editorial assistance. PP acknowledges funding from the Italian Association for Cancer Research (AIRC). LC-C acknowledges funding from the University of California, Davis (Latino Cancer Health Equity Initiative

\section{REFERENCES}

1. Kuchenbaecker KB, Hopper JL, Barnes DR, Phillips KA, Mooij TM, RoosBlom MJ, et al. Risks of breast, ovarian, and contralateral breast cancer for BRCA1 and BRCA2 mutation carriers. JAMA. (2017) 317:2402-16. doi: 10.1001/jama.2017.7112

2. Antoniou AC, Casadei S, Heikkinen T, Barrowdale D, Pylkäs K, Roberts J, et al. Breast-cancer risk in families with mutations in PALB2. N Engl J Med. (2014) 371:497-506. doi: 10.1056/NEJMoa1400382

3. Easton DF, Pharoah PD, Antoniou AC, Tischkowitz M, Tavtigian SV, Nathanson KL, et al. Gene-panel sequencing and the prediction of breastcancer risk. $N$ Engl J Med. (2015) 372:2243-57. doi: 10.1056/NEJMsr15 01341

4. Schmidt MK, Hogervorst F, van Hien R, Cornelissen S, Broeks A, Adank MA, et al. Age- and tumor subtype-specific breast cancer risk estimates for CHEK2*1100delC carriers. J Clin Oncol. (2016) 34:2750-60. doi: 10.1200/JCO.2016.66.5844

5. Shimelis H, LaDuca H, Hu C, Hart SN, Na J, Thomas A, et al. TripleNegative breast cancer risk genes identified by multigene hereditary cancer panel testing. J Natl Cancer Inst. (2018) 110:855-62. doi: 10.1093/jnci/djy106

6. Kiiski JI, Pelttari LM, Khan S, Freysteinsdottir ES, Reynisdottir I, Hart $\mathrm{SN}$, et al. Exome sequencing identifies FANCM as a susceptibility gene for triple-negative breast cancer. Proc Natl Acad Sci USA. (2014) 111:15172-7. doi: 10.1073/pnas.1407909111

7. Peterlongo P, Catucci I, Colombo M, Caleca L, Mucaki E, Bogliolo $\mathrm{M}$, et al. FANCM c.5791C > T nonsense mutation (rs144567652) induces exon skipping, affects DNA repair activity and is a familial breast cancer risk factor. Hum Mol Genet. (2015) 24:5345-55. doi: 10.1093/hmg/ ddv251

8. Neidhardt G, Hauke J, Ramser J, Groß E, Gehrig A, Müller CR, et al. Association between loss-of-function mutations within the FANCM gene and Dean's Fellowship in Precision Health Equity), from the California Initiative to Advance Precision Medicine and from the National Cancer Institute of the National Institutes of Health (Cancer Center Support Grant, P30CA093373). This content is solely the responsibility of the authors and does not necessarily represent the official views of the National Institutes of Health.

and early-onset familial breast cancer. JAMA Oncol. (2017) 3:1245-8. doi: 10.1001/jamaoncol.2016.5592

9. Couch FJ, Nathanson KL, Offit K. Two decades after BRCA: setting paradigms in personalized cancer care and prevention. Science. (2014) 343:1466-70. doi: 10.1126/science. 1251827

10. Michailidou K, Lindström S, Dennis J, Beesley J, Hui S, Kar S, et al. Association analysis identifies 65 new breast cancer risk loci. Nature. (2017) 551:92-4. doi: 10.1038/nature24284

11. Milne RL, Kuchenbaecker KB, Michailidou K, Beesley J, Kar S, Lindström S, et al. Identification of ten variants associated with risk of estrogen-receptornegative breast cancer. Nat Genet. (2017) 12:1767-78. doi: 10.1038/ng.3785

12. Mavaddat N, Michailidou K, Dennis J, Lush M, Fachal L, Lee A, et al. Polygenic risk scores for prediction of breast cancer and breast cancer subtypes. Am J Hum Genet. (2019) 1:21-34. doi: 10.1016/j.ajhg.2018.11.002

13. Spurdle AB, Healey S, Devereau A, Hogervorst FB, Monteiro AN, Nathanson $\mathrm{KL}$, et al. ENIGMA-evidence-based network for the interpretation of germline mutant alleles: an international initiative to evaluate risk and clinical significance associated with sequence variation in BRCA1 and BRCA2 genes. Hum Mutat. (2012) 33:2-7. doi: 10.1002/humu.21628

Conflict of Interest Statement: The authors declare that the research was conducted in the absence of any commercial or financial relationships that could be construed as a potential conflict of interest.

Copyright (C) 2019 Peterlongo and Carvajal-Carmona. This is an open-access article distributed under the terms of the Creative Commons Attribution License (CC BY). The use, distribution or reproduction in other forums is permitted, provided the original author(s) and the copyright owner(s) are credited and that the original publication in this journal is cited, in accordance with accepted academic practice. No use, distribution or reproduction is permitted which does not comply with these terms. 